\title{
PERCEPCIÓN DEL CONCEPTO DE DIGNIDAD HUMANA EN LOS ESTUDIANTES DE LA UNIVERSIDAD PEDAGÓGICA Y TECNOLÓGICA DE COLOMBIA, SEDE DUITAMA
}

\section{PERCEPTION OF THE HUMAN DIGNITY CONCEPT IN STUDENTS AT UNIVERSIDAD PEDAGÓGICA Y TECNOLÓGICA DE COLOMBIA, DUITAMA BRANCH}

\author{
William Ernesto Condiza Plazas 1
}

\section{Resumen}

La investigación se pregunta desde la teoría crítica de los derechos humanos por el significado atribuido a la dignidad humana, por parte de estudiantes universitarios. La investigación se realizó como un estudio transversal descriptivo mixto con dos fases: fase cuantitativa que caracterizó a los estudiantes universitarios de la UPTC sede Duitama con una muestra de 330 participantes, en edades promedio de 21 años. La fase cualitativa buscó comprender los significados atribuidos a la dignidad por los estudiantes universitarios de la sede Duitama, mediante el desarrollo de 7 grupos focales, con la participación de 56 estudiantes analizados por unidades de sentido: dignidad, universidad, vivir como quiera, vivir bien, vivir sin humillaciones. Para los participantes, la dignidad humana significa conocer, ejercer, gozar, ampliar su valor como seres humanos, al mismo tiempo que disfrutar de garantías para el ejercicio de sus derechos humanos. Por su parte, la universidad como institución significa un escenario que propicia la dignidad humana, siempre y cuando sean los seres humanos el centro de su función. El desarrollo de esta investigación contribuyó a la formación integral de los participantes al escuchar, discutir y socializar los diferentes significados atribuidos a la dignidad humana en relación con la universidad.

Palabras clave: dignidad humana, universidad, derechos humanos, respeto.
Recibido: marzo 11 de 2014 Aceptado: junio 20 de 2014
This research wonders from the critical theory of the human rights about the meaning assigned to human dignity based on university students views. It is done as a descriptive cross-sectional mixed study done in to two phases: Firstly, the qualitative phase was characterized by the UPTC students, Duitama Branch, taking part 330 participants under an average age of 21. This phase looked for understanding the meanings given by them to the concept of dignity throughout the development of seven focus groups where 56 university students were analyzed under these meaningful units: dignity, university, living preferences, good living, living without humiliation.

For participants, human dignity means to know, to exercise human right, to enjoy human right, to increase their value as human beings, while enjoying guarantees for the exercise of their human rights. meanwhile, the university as an institution means a setting that fosters human dignity, whenever human beings are the centre of their function. The development of this research contributes to the overall education of the participants, when listening, socializing and discussing the different meanings attributed to human dignity in relation to the university.

Key words: human dignity, university, human rights, respect.

\footnotetext{
${ }^{1}$ Magister en Derechos Humanos. Sociólogo de la Universidad Nacional de Colombia. Profesor Asistente Universidad Pedagógica y Tecnológica de Colombia.Grupo de investigación socio humanística y grupo Símiles. E-mail: condiza@gmail.com
} 


\section{Introducción}

Estudiar la dignidad en relación con la universidad, significa comprender la construcción compleja entre seres humanos que la integran, sus propias prácticas, experiencias, significados y propósitos; es a la vez un esfuerzo por percibir, de manera directa, como se configura el entramado de relaciones que construyen la universidad como institución. Profundizar en la comprensión de estas relaciones desde una teoría crítica de derechos humanos, es un intento por contribuir con preguntas y respuestas, al tiempo que la teoría desarrolla su contenido como praxis, al permitir encontrar la voz de quienes integran la universidad, en este caso, la voz del estudiante universitario.

Por lo tanto, lo que se intenta con la investigación, es por un lado, acercarse a los significados atribuidos a la dignidad por parte de estudiantes universitarios, por ser quienes aportarán en la construcción del futuro de la sociedad colombiana, con su visión comprensiva del mundo, y por otro lado, la necesidad de avanzar y profundizar pluralidades impuras, dotadas de contexto, expresadas en culturas de la dignidad, que se instituyan en culturas diversas de los derechos humanos, indispensables hoy para la supervivencia y la emancipación de millones de seres humanos que padecen la indiferencia del actual sistema mundo.

¿Por qué estudiar la dignidad en relación con la universidad? Entre otras razones, la dignidad humana es el eje de gravitación entre seres humanos e instituciones; además, la universidad es hoy fundamental en el desarrollo del florecimiento humano (Levitas, 2007). A su vez, la "dignidad humana desempeña la función de un sismógrafo que registra lo que es constitutivo de un orden democrático legal, a saber: "precisamente aquellos derechos que los ciudadanos de una comunidad política deben concederse a sí mismos si son capaces de respetarse entre sí, como miembros de una asociación voluntaria entre personas libres e iguales" (Habermas, 2010, p. 10), en cuya constitución solo es posible sí entre iguales se exige tratar a todas las personas como un fin en sí mismo (Kant, 2003).

Para Kant, "la humanidad misma es dignidad: porque el hombre no puede ser utilizado únicamente como medio por ningún hombre (ni por otros, ni siquiera por sí mismo), sino siempre a la vez como fin, y en esto consiste precisamente su dignidad (la personalidad) en virtud de la cual se eleva sobre todas las cosas" (1989. p. 335). Reconocer lo anterior es necesario para que los integrantes de la comunidad universitaria puedan vivir en sociedad, con la riqueza de una cultura de la dignidad, esto es, una cultura que armonice al ser humano con la naturaleza, y una cultura de la dignidad humana, es decir, una cultura que reconoce las diferencias y pluralidades del ser humano, que construye su significado en contextos impuros, dotados de necesidades que han de ser satisfechas por mecanismos concretos, en un espacio y tiempo dado. La dignidad humana como núcleo de las relaciones sociales permite conocer, ejercer, gozar, ampliar y disfrutar los derechos humanos.

Es por lo tanto, un compromiso ético y político por encontrar el significado atribuido a la dignidad por seres humanos que construyen la universidad como institución de educación superior, y que a su vez, asumen su responsabilidad de formarse como seres humanos integrales, con capacidades y potencialidades integradoras, críticos, propositivos, más allá de ideas productivistas (la instrucción-"educación" para la producción), o de buenos ciudadanos (la instrucción-educación para la obediencia).

La universidad más allá de un espacio de generación de conocimiento, es además, una institución que permite el encuentro de diferentes formas de ser y existir, por esta razón, preguntarse por la dignidad en relación con la universidad, es también preguntarse por el reconocimiento de lo 
heterogéneo, por reconocer que diferentes formas de ser y estar en el mundo, no solo son "utopía", sino que corresponden a la diversidad que nos hace humanos; es por ello que la universidad es un espacio plural y multiverso (véase el trabajo de Krotz, 2003).

De acuerdo con Sarmiento (2013), damos por "supuesto" que el ser humano es un ser dotado de dignidad intrínseca, en cuanto ser auto reflexivo, consiente, libre, sujeto social, perteneciente a una especie biológica y universal, que mediante el trabajo, actividad vital, libre y creativa, transforma la naturaleza y lo constituye en la historia como ser humano, pero, ¿qué es la dignidad para quienes integran la universidad, cuales son los significados atribuidos a la dignidad?, ¿es la universidad una institución que permite la realización de la dignidad?. Esta investigación se limita a estudiar el significado de la dignidad humana en estudiantes universitarios; sin embargo, es necesario incluir para próximos trabajos, el significado atribuido a la dignidad humana por parte de otros integrantes de la comunidad universitaria: profesores, administrativos y demás actores que integran la universidad.

Desde el enfoque de universidad saludable se han desarrollado innumerables publicaciones y contribuciones a la descripción de los estudiantes universitarios (Machado, 2001); también se los ha descrito para dar sentido a los movimientos y trasformaciones sociales del siglo XX (Hobsbawm, 1998). Por otra parte, los estudios de juventud, incluyen a los estudiantes universitarios preguntándose por los cambios sociales, políticos, históricos y culturales en razón a la vida cotidiana de esta población (Sánchez, 2006); también se han adelantado trabajos para observar el consumo de sustancias psicoactivas por diferentes agencias gubernamentales, en el contexto próximo, destacándose los trabajos de la Comunidad Andina de Naciones (CAN, 2013), cuyas principales conclusiones refieren al estudiante universitario en permanente situación de riesgo en diferentes ámbitos y particularmente como potencial consumidor de sustancias psicoactivas. De otro lado, los trabajos que abordan la dignidad humana como problema de investigación y los significados que los estudiantes universitarios construyen, son escasos.

La dignidad ha sido más un tema del derecho, la filosofía, la teología, no para escuchar al estudiante universitario, y otros saberes igualmente importantes, como el significado atribuido a la dignidad humana, para y por las mujeres, las personas en situación de discapacidad, las comunidades indígenas, las comunidades LGTBI, las comunidades campesinas, las personas afrodescendientes, entre muchos otros, o en palabras de Michel Foucault (1978) "los otros", con los que nadie cuenta, sino, para darle fundamento al desarrollo teórico de estas áreas.

Trabajos como el de Carrillo (2011), intentan acercarse a lo que el autor denomina el espíritu universitario y autonomía; la preocupación por la dignidad en esta obra está asociada tácitamente al desarrollo de la universidad como un conjunto de personas - hombres y mujeres-, con libertad, con dignidad, que aspiran a acercarse a la verdad, al bien, a la belleza, a la justicia (Carrillo, 2011 p. 9), sin embargo, sigue siendo el autor quien habla y quien construye sus concepciones. Para Gómez (2011) la dignidad de la persona es el fundamento del orden jurídico y de la teoría del sujeto de derecho penal; de acuerdo con este autor, la dignidad se erige como el principio de los principios y la norma que norma todo lo jurídico; Gómez destaca la importancia de la dignidad, pero nuevamente su esfuerzo deja por fuera el escuchar a "los otros", y de esta manera, cuando se habla de dignidad humana, solo hablan los especialistas, en abstracto: filósofos, abogados y teólogos, pero ¿qué es la dignidad humana, para las comunidades en concreto?, ¿por qué frente a la opresión muchas comunidades tienen como bandera la dignidad y como horizonte los derechos humanos?, estas son preguntas que requieren un programa fuerte de investigación 
que se escapa a los propósitos del presente artículo, no obstante, escuchar a los estudiantes es una manera de dignificar a"los otros".

Para la teoría crítica la dignidad humana es eje de gravitación de los derechos humanos (Restrepo, 2010), y el valor intrínseco que permite al ser humano luchar incansablemente por su lugar en el mundo; para esta teoría la dignidad, además, permite ampliar los derechos humanos al encontrar nuevas formas de ser y de existir en el mundo, es así que la dignidad humana, se profundiza y se dinamiza. Desde esta perspectiva la dignidad humana no es solo concepto filosófico, ni un principio, ni una norma, es una lucha permanente por la humanización y la relación armónica con la naturaleza. Para esta teoría, en ausencia de dignidad humana no existe el ser humano, por tal razón es fundamental dar la palabra a "los otros", que en otras palabras, es "ese nosotros" que nos configura como seres humanos con sueños, deseos, anhelos, necesidades, miedos, etc.

Hablar de dignidad nos obliga a encontrar caminos que conduzcan a su construcción, no como algo dado a priori, intrínseco al ser humano, sino como lucha permanente por hacernos humanos, lo cual se hace posible con ayuda del análisis crítico, en donde escuchándonos los unos a "los otros", reconociendo nuestra diversidad implícita en tanto consecuencia de pluralidadcomplejidad de visiones comprensivas del mundo, formas de ser y estar, propuestas de futuro, entre muchos otros aspectos, podremos encontrar los significados atribuidos a la dignidad humana, fundamentales para ejercer derechos humanos, construir sociedad, y vivir en paz.

Posterior a la exposición del método, el artículo presenta sus resultados en tres momentos: i) descripción de aspectos sociodemográficos de los participantes que permite establecer su perfil, ii) aproximación a la dignidad humana que establece algunos significados asociados, iii) consideraciones sobre la universidad en general, teniendo en cuenta los hallazgos.

\section{Método}

\subsection{Tipo de estudio y población}

La investigación se realizó como un estudio transversal descriptivo mixto con dos fases: cuantitativa y cualitativa. La población estuvo constituida por estudiantes de la Universidad Pedagógica y Tecnológica de Colombia, UPTC, Facultad Seccional Duitama, pertenecientes a los siguientes programas académicos: Administración de Empresas Agropecuarias, Administración Industrial, Administración Turística y Hotelera, Diseño Industrial, Ingeniería Electromecánica, Licenciatura en Educación Industrial, Licenciatura en Matemáticas y Estadística (la información del porcentaje de participación y semestre se expone en la sección de resultados).

Se plantearon dos grupos de participantes: el primer grupo, compuesto por quienes dieron respuesta a la encuesta; El tipo de muestreo fue el muestreo teórico o intencionado, que tuvo inicio mediante voluntarios y posteriormente mediante proceso de avalancha, avanzando hacia una estrategia de muestreo deliberado basado en las necesidades de información detectadas (Salamanca y Martín-Crespo, 2007), considerándose una muestra de 330 estudiantes.

El segundo grupo participó en la fase cualitativa del estudio a través de siete grupos focales convocados de manera intencional, conformados por 56 estudiantes, a quienes se les preguntó por su percepción del concepto de dignidad humana. Los grupos focales fueron grabados y analizados por las siguientes unidades de sentido: dignidad, universidad, vivir como quiera, vivir bien y vivir sin humillaciones.

Posteriormente se construyó una lista de 100 palabras asociadas a dignidad, derivadas de los grupos focales, la cual fue calificada a través de la 
aplicación formularios de google. La investigación se desarrolló entre los meses de agosto y noviembre de 2013.

\subsection{Instrumentos}

Para realizar la investigación se emplearon dos instrumentos. El primero denominado: caracterización del perfil de los estudiantes de Ciencias Humanas de la Universidad Nacional de Colombia, elaborado por docentes encargados del áreas salud, psicosocial y socioeconómica, dirigido por la PhD Patricia Jaramillo, en el año 2001. El cuestionario fue adaptado para los objetivos del proyecto de investigación universidad y dignidad, que incluyó preguntas abiertas. El instrumento consta de las secciones: sociodemográfico, socioeconómico, vivienda, motivaciones y expectativas, salud, grados de afectación de diferentes situaciones, aspectos políticos, y representaciones sociales. Esta presentación como análisis preliminar y parcial de la información, tiene en cuenta los aspectos sociodemográfico, socioeconómico, y políticos.

El segundo instrumento denominado: protocolo de grupo focal, que incluía las categorías: dignidad, plan vital, vivir sin humillaciones y condiciones materiales; se adaptó y agrupó en las categorías emergentes "vivir como quiera", "vivir bien", "vivir sin humillaciones" y posteriormente se incluyó una lista con 100 palabras que los participantes asociaron con la dignidad humana.

\subsection{Procedimiento}

La primera fase, cuantitativa, se realizó mediante la aplicación de una encuesta que buscaba caracterizar a los estudiantes universitarios de la UPTC sede Duitama; la recolección de información se llevó a cabo durante un mes de manera individual y autoaplicada empleando como herramienta, el servicio de encuestas de la plataforma de google; la información fue analizada mediante estadísticos descriptivos usando el paquete estadístico SPSS 19, discriminando por sexo, edad y semestre. Antes de diligenciar el cuestionario, los participantes fueron informados sobre la confidencialidad de sus respuestas y el manejo ético de la información.

La segunda fase, cualitativa, buscó comprender los significados atribuidos a la dignidad, por parte de los estudiantes universitarios en la sede Duitama mediante el desarrollo de 7 grupos focales analizados por unidades de sentido; los estudiantes fueron informados sobre los objetivos y manejo confidencial de la información, firmando consentimiento informado.

\section{Resultados}

\section{Aspectos Sociodemográficos de los Participantes}

De los 330 participantes en la investigación, 24\% correspondió a la carrera de Administración de Empresas Agropecuarias, 18\% Administración Industrial, 12\% Administración Turística y Hotelera, $9 \%$ a la carrera de Diseño Industrial, 14\% a Ingeniería Electromecánica, 7\% a la carrera de Licenciatura en Educación Industrial, 12\% a la carrera de Licenciatura en Matemáticas y Estadística y finalmente $5 \%$ a la carrera de Licenciatura en Tecnología. En la tabla 1 se presenta el porcentaje de participación por semestre.

\begin{tabular}{cc}
\hline Semestre & Porcentaje de participación \\
\hline I Semestre & $16 \%$ \\
II Semestre & $15 \%$ \\
III Semestre & $8 \%$ \\
IV Semestre & $7 \%$ \\
V Semestre & $8 \%$ \\
VI Semestre & $9 \%$ \\
VII Semestre & $9 \%$ \\
VIII Semestre & $11 \%$ \\
IX Semestre & $9 \%$ \\
X Semestre & $4 \%$ \\
Mayor XI Semestre & $6 \%$ \\
\hline
\end{tabular}

Tabla 1. Participación de estudiantes por semestre

El 54\% de los participantes reportó ser del sexo masculino y el $46 \%$ de las participantes de sexo femenino. La edad promedio de los participantes 
es 21 años, encontrándose participantes en el rango de 15 a 50 años. Proceden del municipio de Duitama 52\%, de municipios aledaños a Boyacá $33 \%$, de otras partes del país $14 \%$ y del extranjero $1 \%$.

Los participantes reportaron que el colegio donde terminaron su bachillerato era privado en $13 \%$ de los casos, departamental 10\%, nacional 13\%, distrital municipal $29 \%$, femenino $1 \%$, masculino 2\%; mixto 33\%. La institución educativa en el 70\% de los casos no era bilingüe y el $30 \%$ si lo era.

Al preguntarles si tenían pareja, los participantes contestaron: pareja permanente entendida como matrimonio o unión marital de hecho, el 15\% respondió afirmativamente, parcialmente, entendida como noviazgo 33\%, no tiene pareja el $52 \%$. Respecto a si tienen o no hijos contestaron sí el $8 \%$ y no el $92 \%$. Entre quienes tienen hijos, se consultó por su edad, el $75 \%$ dijo que sus hijos son menores de 5 años; entre cinco y 10 años 20\%, y $5 \%$ mayores de 10 años.

Ante la pregunta de la existencia del padre y de la madre, el $2 \%$ de los participantes no sabe si su padre vive, el $90 \%$ de los participantes señalaron que su padre vive, no vive el $8 \%$ de los padres; por su parte, el $95 \%$ de los participantes indicaron que su madre vive, $5 \%$ señalaron que su madre no vive. Al indagar por la convivencia con los padres (padre - madre), entendiéndola como permanente, esporádica, y nula se encontró que ha sido permanente la convivencia con el padre en un $69 \%$, ha sido esporádica $19 \%$, es decir, que ha vivido con el padre por algunos periodos de tiempo; nula $11 \%$. Por su parte, la convivencia con la madre ha sido permanentemente $92 \%$, esporádica $5 \%$ y nula en $3 \%$. Con respecto al nivel educativo alcanzado por los padres, este se puede observar en la tabla 2.

Al preguntar a los participantes qué tipo de relación tiene con su familia, entendiéndose como vivir con la familia y relación económica, $67 \%$ de

\begin{tabular}{lcc}
\hline Nivel Educativo & Padre & Madre \\
\hline Primaria incompleta & $28 \%$ & $26 \%$ \\
Bachillerato & $36 \%$ & $41 \%$ \\
Técnico & $9 \%$ & $12 \%$ \\
Tecnóloqo & $4 \%$ & $6 \%$ \\
Universitario & $12 \%$ & $9 \%$ \\
Postarado & $1 \%$ & $1 \%$ \\
Ninquno & $1 \%$ & - \\
No sabe & $8 \%$ & $4 \%$ \\
Otro & $1 \%$ & $1 \%$ \\
\hline
\end{tabular}

Tabla 2. Nivel educativo padres

El nivel ocupacional de los padres se describe en la tabla 3.

\begin{tabular}{lcc}
\hline Nivel Ocupacional & Padre & Madre \\
\hline Desempleado & $4 \%$ & $2 \%$ \\
Empleado & $40 \%$ & $28 \%$ \\
Independiente & $43 \%$ & $22 \%$ \\
Hoqar & $2 \%$ & $45 \%$ \\
No sabe & $11 \%$ & $3 \%$ \\
\hline
\end{tabular}

Tabla 3. Nivel Ocupacional Padres

los participantes vive con su familia y depende de ellos, $17 \%$ vive con su familia y no depende de ellos, $9 \%$ no vive con su familia y depende de ellos, $7 \%$ no vive con su familia y no depende de ellos. Al preguntarles ¿De quién depende económicamente? los participantes contestaron $42 \%$ padre y madre, $23 \%$ de sí mismo, $16 \%$ solo de la madre, $13 \%$ solo del padre, otro $19 \%$.

A la pregunta ¿Cuántas personas dependen del ingreso familiar? $50 \%$ entre 1 y 3, 42\% entre 4 y 5 , $8 \%$ entre 6 y 8 . De acuerdo a las indagaciones, el ingreso mensual del núcleo familiar se encuentra en los siguientes rangos en pesos colombianos (COP), menos de $\$ 300.000$ COP, $5 \%$; entre $\$ 300.000$ y $\$ 500.000$ COP, $28 \%$; entre $\$ 500.000$ y $\$ 1.000 .000$ COP, $34 \%$; entre $\$ 1000.000$ y $\$ 1.500 .000$ COP, $13 \%$; entre $\$ 1.500 .000$ y $\$ 2.000 .000 \mathrm{COP}, 9 \%$; entre $\$ 2.000 .000$ y $\$ 2.500 .000$ COP, $6 \%$; entre $\$ 2.500 .000$ y $\$ 3.000 .000$ COP, 3\%; entre $\$ 3.500 .000$ y $\$ 4.000 .000$ COP, $2 \%$; más de $\$ 4.500 .000$ COP, $2 \%$.

Al preguntarles ¿Cuál es el estrato socio económico de su lugar de residencia? Los participantes contestaron estrato 1: $8 \%$; estrato 2 : 
55\%; estrato $3: 30 \%$; estrato $4: 5 \%$; estrato $5: 1 \%$; no se reporta personas de estrato 6 . Por otra parte, el $1 \%$ de los participantes señala que su estrato es rural. Al preguntar el tipo de propiedad se obtuvieron los siguientes datos: vivienda propia $61 \%$; vivienda en arriendo 34\%; vivienda en hipoteca $2 \%$; en comodato (vivienda asignada como parte de salario integral) $1 \%$; otro $2 \%$, al indagar por este porcentaje es necesario advertir que corresponde a personas que viven en el área rural por una parte, y por otra, personas que disponen de una vivienda bajo la modalidad de empeño.

A la pregunta está trabajando actualmente, el $52 \%$, no lo hace y el $48 \%$ si lo hace, del total de quienes están trabajando solo el $18 \%$ trabaja en un área referida a su campo de estudio, mientras que el $82 \%$ trabaja en áreas diferentes a su área de estudio, obteniendo ingresos inferiores a un salario mínimo. El promedio mensual de los gastos de los participantes es entre $\$ 200.00$ y $\$ 300.000$ COP, $52 \%$; entre $\$ 300.00$ y $\$ 500.000$ COP, 32\%; entre $\$ 500.00$ y $\$ 700.000$ COP, $11 \%$; entre $\$ 700.00$ y $\$ 900.000$ COP, $4 \%$ entre $\$ 900.00$ y $\$ 1.100 .000$ COP, $1 \%$. Siendo los gastos de mayor prioridad: alimentación 27\%; transporte 23\%; pago de matrícula 22\%; vivienda 9\%; fotocopias $8 \%$, comunicación (internet - teléfono) 6\%; esparcimiento y cultura 3\%, Educación (Diferente UPTC) $2 \%$.

En resumen, al hablar del perfil sociodemográfico de los participantes de la investigación, hablamos de una persona de 21 años procedente del municipio de Duitama, que terminó sus estudios de bachillerato en un colegio mixto distrital municipal no bilingüe. Soltero(a). Convive y depende económicamente de sus padres, quienes en su mayoría son bachilleres, en el caso del padre trabajando independientemente o como empleado, y en caso de la madre dedicada al hogar, el grupo familiar dispone de ingresos entre $\$ 500.000$ y $\$ 1.000 .000 \mathrm{COP}$, del cual dependen entre 1 y 3 personas. Los participantes pertenecen en su mayoría al estrato 2, la mayor parte viven en casa propia. El promedio mensual de gastos de los participantes está entre $\$ 300.00$ y $\$ 500.000$ COP, que se destinan a pagar alimentación, transporte, matrícula, vivienda, fotocopias, comunicación (internet - teléfono), esparcimiento, cultura y educación diferente a la UPTC.

\section{Aproximación a la Dignidad humana}

La pregunta de inicio en los grupos focales era ¿Qué es para ti la dignidad humana?, de un total de 140 intervenciones en esta pregunta, los significados que más se asociaron fueron en su orden referido: persona (106 intervenciones) respeto (57 intervenciones), valor (57 intervenciones), derecho (28 intervenciones), ser humano (30 intervenciones) libertad (27 intervenciones), capacidad (16 intervenciones), vivir (15 intervenciones), sentirse (13 intervenciones) orgullo (8 intervenciones), necesidad ( 3 intervenciones), valores (6 intervenciones) igualdad (5 intervenciones), autonomía (4 intervenciones). Aquí se habla de intervenciones para diferenciarlas de porcentajes, dado la unidad de sentido que tiene cada una de las intervenciones, en razón del texto y contexto en el cual las palabras dentro de las intervenciones cobran sentido y significado.

Dentro del cuestionario se pidió a los participantes puntuar de una lista de 100 palabras sugeridas por ellos mismos en los grupos focales, que a su parecer más asociaran con el significado de dignidad humana, obteniendo los resultados que se presentan en la tabla 4.

\begin{tabular}{|c|c|c|c|c|c|}
\hline & Mucho & Bastante & Medianamente & Poco & Muy poco \\
\hline Respeto & $71 \%$ & $26 \%$ & $2 \%$ & $0 \%$ & $1 \%$ \\
\hline Autoestima & $63 \%$ & $25 \%$ & $1 \%$ & $5 \%$ & $6 \%$ \\
\hline Derecho & $58 \%$ & $29 \%$ & $5 \%$ & $3 \%$ & $5 \%$ \\
\hline Felicidad & $57 \%$ & $20 \%$ & $18 \%$ & $3 \%$ & $2 \%$ \\
\hline Libertad & $49 \%$ & $32 \%$ & $14 \%$ & $3 \%$ & $2 \%$ \\
\hline Equidad & $48 \%$ & $29 \%$ & $15 \%$ & $6 \%$ & $2 \%$ \\
\hline Autonomía & $46 \%$ & $32 \%$ & $14 \%$ & $5 \%$ & $3 \%$ \\
\hline Vivir Bien & $46 \%$ & $32 \%$ & $11 \%$ & $8 \%$ & $3 \%$ \\
\hline Amar & $46 \%$ & $25 \%$ & $15 \%$ & $12 \%$ & $2 \%$ \\
\hline Moral & $43 \%$ & $38 \%$ & $14 \%$ & $3 \%$ & $2 \%$ \\
\hline
\end{tabular}

Tabla 4. Palabras asociadas a dignidad humana 
Los participantes asocian la dignidad humana con el respeto como una forma de reciprocidad, señalan que para que la dignidad pueda existir es necesario un alto grado de reconocimiento de los otros, sin embargo, esto no es posible si no existe autoestima, que para los participantes más que ser una forma de evaluación del valor de cada uno, es más comprendida como capacidad de ser.

Al derecho lo asocian con la dignidad en tres planos, el primero con lo jurídico, comprendido como una lista de normas y deberes que "se deben cumplir", conocer y cambiar-actualizar; el segundo con los derechos humanos, que para los participantes no son posibles sin dignidad humana. Por otra parte, al hablar de derechos humanos no es claro para los participantes, el ejercicio de los derechos humanos; en este punto la investigación, sin proponérselo, cumplió un papel de pedagogía de los derechos humanos, al facilitar un intercambio intersubjetivo entre el investigador y los participantes. El tercer plano lo denominaron el plano "personal" donde, según la mayoría de los participantes, es necesario saber a dónde acudir en caso de violación de sus derechos; aquí el derecho se entiende como algo externo que debe ser provisto por una institución, similar a un servicio; para un próximo análisis será necesario profundizar en la distorsión que genera el neoliberalismo sobre los derechos.

Para los participantes, la felicidad es una aspiración permanente, no en el futuro sino en el presente y está muy asociada con las ideas de universidad, proyecto vital y vivir bien. La felicidad también es para los participantes una lucha por no perder la esperanza y construir permanentemente sus ambiciones, deseos, anhelos, y voluntades. Un acto de felicidad dentro de la universidad se asocia con el cumplimiento de metas y expectativas: obtener "buenas calificaciones", ganar en una competencia deportiva, salir de práctica académica y conocer nuevas personas, entre otros aspectos asociados.

La libertad, al asociarla con la dignidad, es para los participantes un ejercicio de respeto, en el que la voluntad está sujeta a no maltratar, dañar o menospreciar a otra persona. Por su parte, la autonomía para los participantes, es el reconocimiento "que en el mundo no se está solo" y que por lo tanto, es necesario imponerse algunos límites pero romper otros. Hablan de la salida de su hogar actual, como un ejercicio de la autonomía, que podrán realizar al terminar sus estudios.

La equidad para los participantes es un ejercicio de justicia, percibida como armonía entre las personas y la sociedad, como una forma de igualdad, en la que la ley es la que nos hace equiparables, pero no iguales. En los grupos focales al hablar de equidad llamó mucho la atención del investigador que otra asociación fuerte aparte de la dignidad tiene que ver con lo que denominan "los menos favorecidos", es decir para los participantes equidad es sinónimo de asistir.

Vivir bien para los participantes es poder ser libres, felices, logrando ejercer sus derechos, estableciendo su voluntad, viviendo sin humillaciones. Al preguntar a los participantes ¿qué es para ustedes vivir bien?, su preocupación central se orientaba, en la mayoría de los casos, al desarrollo de su carrera como un medio que les proveerá de los recursos económicos necesarios para cumplir sus expectativas, entre los que se destaca: tener una familia, viajar y disponer con medios materiales suficientes.

Amar, al asociarlo con dignidad, para los participantes es primero felicidad, segundo autonomía, tercero autoestima, cuarto una asociación con libertad. Expresan que para amar es necesario poder encontrar una persona después de haber compartido con otras personas que los preparen para conocer el amor. El amor es para ellos un misterio al que no conocen bien y que esperan vivir "más adelante". Otra asociación, contraria a la idea de libertad es la idea de dependencia y falta de autonomía, en la que el "ser amado" podría dificultar o frustrar expectativas, 
"sueños", anhelos o deseos.

Ahora se toman tres intervenciones, de forma textual, para referirnos al significado atribuido a la dignidad humana por los participantes. Estas intervenciones fueron seleccionadas teniendo en cuenta el consenso generado en los grupos focales, lo que a su vez sirve como estrategia de cointerpretación entre participantes e investigador (el número de las intervenciones corresponde al reglón de transcripción): Primera intervención (Intervención 29):"la dignidad es la capacidad que tiene una persona para vivir bien. La dignidad es el respeto que debemos tener hacia las demás personas y la igualdad entre uno y otro respetarse a símismo, valorarse".

Segunda intervención (Intervención 101): “Darse un lugar, la dignidad es vivir en condiciones que puedan satisfacer las necesidades humanas según la escala, respeto propio capacidad de defender los principios hasta las últimas consecuencias. La dignidad es el valor que tiene una persona por sus acciones, comportamientos, formas de ser. Equivale a respeto".

Tercera intervención (Intervención 83): "dignidad es amarse".

Teniendo en cuenta lo anterior se puede afirmar que, la dignidad humana significa conocer, ejercer, gozar, ampliar su valor como seres humanos, al mismo tiempo que disfrutar de garantías para el ejercicio de sus derechos humanos. La dignidad para los participantes es sinónimo de respeto, de darse un lugar y de amar a los otros y así mismo, de ser reconocidos como valiosos, de vivir bien, y sin humillaciones y a la vez contar con las condiciones físicas, mentales y los medios necesarios para realizar su plan vital o su proyecto de vida.

Una definición similar se encuentra en Sarmiento (2013), para quien la dignidad humana es aquella condición especial que reviste a todo ser humano, por el hecho de serlo, y lo caracteriza de forma permanente y fundamental desde su concepción hasta su muerte, más aún, lo trasciende. La cualidad de digno hace referencia al valor inherente al ser humano en cuanto despliegue multilateral de sus fuerzas ontológicas que lo constituyen y desarrollan en la historia, por tanto, la dignidad es el valor supremo de la humanidad entendida como especie social universal (Sarmiento, 2013). Por su parte Herrera señala que "la dignidad consiste en la obtención de un acceso igualitario a los bienes tanto materiales como inmateriales que se han ido consiguiendo en el constante proceso de humanización del ser humano" (2008. p. 108), entonces, para que la dignidad humana sea posible es necesario que todos los seres humanos cuenten con los instrumentos (legales, jurídicos, políticos etc.), medios (estados construidos sobre la protección del ser humano y no del mercado, por ejemplo) y condiciones necesarias para poder poner en práctica la dignidad humana como ejercicio de una vida buena con el desarrollo de capacidades, potencialidades, autonomía, solidaridad, armonía (multiculturalidad, educación, democracia real, participación real, etc.).

Al aproximarnos al significado de dignidad humana asociado con los derechos humanos, los participantes comparten parcialmente -existe una equiparación entre derecho y servicio-, que los derechos humanos no son un ideal abstracto, que corresponde a una racionalidad del cálculo, no son un servicio el cual un agente del mercado los satisface, tampoco son ese algo vacío de la lógica neoliberal, carente de significado, en nombre del cual se hace la guerra o se defiende a la humanidad buena de la humanidad mala, de acuerdo al interés con que se analice. Por el contrario, los derechos humanos en concreto "son el conjunto de procesos que los individuos y los grupos humanos ponen en marcha cotidianamente para acceder a los bienes exigibles para una vida digna de ser vivida, es decir, son una permanente lucha por la dignidad humana"(Herrera, 2008 p .108). Los participantes permanentemente discutieron la necesidad de contar con los medios tangibles e intangibles necesarios para poder disfrutar de la 
vida buena, a la que asocian la dignidad humana, los derechos humanos y la universidad como una institución que debe garantizar un escenario propicio para construir dignidad.

\section{Universidad}

Al preguntar a los participantes ¿Si nuevamente tuviera la oportunidad de escoger universidad y carrera usted elegiría?, los participantes contestaron: la misma universidad y la misma carrera 55\%, la misma universidad y distinta carrera $25 \%$, diferente universidad y diferente carrera $11 \%$, diferente universidad y la misma carrera 9\%. Al asociar con la pregunta ¿Cuáles fueron las razones más importantes que lo llevaron a escoger la UPTC? Los participantes señalan como principal razón los costos económicos con $34 \%$, casi con el mismo porcentaje la influencia familiar o de algún conocido $21 \%$, formación académica de alto nivel $20 \%$, prestigio y tradición de la institución $19 \%$, no tuvo otra opción 7\%. Por su parte, las razones de elección de carrera están asociadas a gusto, o atracción hacia la carrera 29\%, alto rendimiento en un área similar en el colegio 13\%, influencia de algún familiar o de algún conocido $12 \%$, vocación $11 \%$, posible remuneración económica laboral alta $10 \%$, prestigio social $6 \%$, lograr pasar en la universidad $6 \%$, orientación profesional en el colegio $5 \%$, motivaciones altruistas-intelectuales $4 \%$, no tuvo otra opción $4 \%$.

El grado de satisfacción con la universidad, el programa curricular, la infraestructura de la Universidad y los costos educativos se resumen en la tabla 5.

\begin{tabular}{lcccc}
\hline \multicolumn{1}{c}{$\begin{array}{c}\text { Grados de } \\
\text { satisfacción }\end{array}$} & Universidad Programa & Infraestructura Costos \\
\hline Satisfecho & $52 \%$ & $40 \%$ & $8 \%$ & $43 \%$ \\
Satisfacción parcial & $46 \%$ & $55 \%$ & $53 \%$ & $46 \%$ \\
Insatisfecho & $2 \%$ & $5 \%$ & $39 \%$ & $11 \%$ \\
Total & $100 \%$ & $100 \%$ & $100 \%$ & $100 \%$ \\
\hline
\end{tabular}

Tabla 5. Grados de satisfacción

También se indagaron las razones que llevan a seguir estudiando en la UPTC, obteniendo como resultado: mejorar calidad de vida $21 \%$, realizarse intelectualmente $17 \%$, terminar la carrera $15 \%$, realizarse laboralmente $15 \%$, satisfacción económica $12 \%$, crear una empresa $10 \%$, mejorar el bienestar colectivo $4 \%$, prestigio status social $3 \%$, dedicarse a la investigación $2 \%$. Llama la atención que en su mayoría los propósitos de los participantes son de carácter individual y están asociados con temas económicos; preocupa que la investigación solo interese al $2 \%$ de los participantes.

Los participantes consideran a la universidad como una institución que propicia la dignidad al permitirles desarrollar parte de su plan vital, terminar una carrera no es lo único que los motiva, insisten en la cooperación entre pares a la hora de cursar la carrera y egresar. Además, le otorgan a la universidad importancia al proveerles conocimiento que puede ser aprovechado en el beneficio individual. Igualmente, los participantes comentan lo importante que es la universidad para ellos como un espacio lúdico y artístico que permite el desarrollo integral de habilidades.

Están preocupados por discursos que distorsionan o simplifican el papel de la universidad en la sociedad (Véase León, 2005), la universidad productivista basada en las competencias y el desarrollo del "egoísmo" sin espacio ni tiempo, alejada de los problemas que consideran importantes es para ellos fuente alarma.

La universidad como institución para los participantes, significa un escenario que propicia la dignidad humana, siempre y cuando sean los estudiantes reconocidos como seres humanos el centro de su función y no las demandas del mercado, que transforman la naturaleza de la universidad como lo describe Noam Chomsky cuando afirma que "el neoliberalismo tomó por asalto a las universidades", para el autor "el modelo empresarial en el que tienden a inscribirse las instituciones de educación superior precariza la calidad de la enseñanza y el aprendizajey 
reproduce dinámicas autoritarias indeseables para las sociedades actuales" (Chomsky citado por Mendoza, 2014).

De otro lado, la imagen subjetiva que los actores se hacen (Giddens, 2000), permite representar a la universidad como un sistema en continua interacción entre los individuos que integran la institución. Desde este ángulo, hay una permanente construcción en la que se enfatiza la incertidumbre, la impredictibilidad, la inestabilidad y la complejidad institucional. Relación entre estructuras, imágenes y culturas académicas que condiciona la permanencia en la universidad y puede hacer de ella una experiencia difícil. El ambiente de la universidad, para el estudiante, por su particular riqueza de miradas y complejidad en sus formas de vida, así como por la dinámica propia que le imprime esta misma riqueza, es un escenario que puede ser generador de grandes problemas o por el contrario un contexto que permita la dignificación y de esta manera le permita encontrar respuestas a las múltiples inquietudes que suscita la vida en el mundo contemporáneo.

La universidad es un escenario con características que la hacen única, tanto por quienes la conforman, como por los importantes recursos de carácter cognitivo y el considerable nivel de exigencia demostrados en el ingreso y permanencia en la universidad. Es por ello un contexto en el que la exposición a crisis existenciales, angustias, incertidumbres y presiones ocasionadas por expectativas diversas, que a su vez, pueden revertirse en depresiones y otros trastornos que dificulten la realización de la dignidad humana entendida para ellos como bienestar y un futuro en diferentes aspectos como sinónimo de"vida estable".

El estudiante universitario está sujeto, en muchos casos, a imaginarios en torno al éxito académico, ligados a un futuro laboral exitoso, que en el contexto actual del mercado laboral colombiano de precarización del empleo, des-laboralización y reducción paulatina del estado social de derecho, dicho imaginario, es decir, "entrar a la universidad para conseguir un empleo bien remunerado" y de esta manera trabajar para el acenso social cada vez es menos probable.

La toma de decisiones en el mundo contemporáneo implica exposiciones al riesgo y a la incertidumbre. Una de las decisiones más difíciles que tiene que tomar una persona en el trascurso de su vida, se refiere a la elección vocacional, la carrera a la que probablemente se dedicará toda su vida. Es una de las pocas decisiones en las cuales no se puede fallar, pues el costo de "equivocarse de carrera" puede llegar a ser muy alto. Además, es necesario tener en cuenta la presión ejercida por las condiciones laborales que pueden en muchos casos determinar la elección de la carrera y la satisfacción lograda con esta decisión. De allí el valor otorgado al rendimiento y ponderado a través de las notas, sea convertido en un indicador de la efectividad de la decisión tomada. Ante la ausencia de una prueba fehaciente de sentirse en el lugar correcto al haber ingresado a la universidad, así como de poder desempeñarse en el futuro con lo que se ha aprendido, las calificaciones se convierten en la mejor prueba. Uno de los hitos de ese proceso de construcción individual es justamente la definición vocacional, y con ella, muchas de las decisiones tomadas durante el período de permanencia en la universidad. Este período es para muchos jóvenes, el tránsito hacia la adultez. La definición vocacional implica el constante cuestionamiento frente a la habilidad individual ante el desempeño académico y normalmente se acompaña de la adopción de un sentido de vida (Hernández 2007).

La búsqueda de sentido para la vida es un problema social generalizado (Luhmann, 1996), se expresa en la aparición de un sin número de neogrupos que buscan contener como una institución total al ser humano, tratando de cercar su vida, a 
cambio de proveerle certidumbre (Berger, 1997), al sentirse parte de. Sin embargo, la misma cantidad de ofertas con ideas tan plurales hacen que la incertidumbre se renueve y se masifique, ya nada está dado para quien empieza en la Universidad, ni para quien sale de ella, y preguntas llenas de angustia como ¿Si existe vida después del pregrado?, son cada vez más recurrentes si no se tiene la "certidumbre vocacional", minada por la emoción permanente de soledad al tener que tomar decisiones con información imperfecta.

La soledad para este grupo de estudiantes, significa un riesgo, que deben asumir en su proceso de formación. Parra (1994) ha señalado que la soledad es cada vez más latente en la universidad, al ser esta una institución masiva, sin embargo, para los participantes, la soledad es más una condición necesaria para construir su autonomía, y vivir dignamente, entonces la soledad para este grupo de estudiantes no tiene ese carácter negativo que Sandoval enfatiza.

Finalmente, al anterior panorama se suma lo descrito en el trabajo de los ingenieros Frey, y Osborne, (2013), titulado: The future of employment, quienes a través de un sofisticado análisis estadístico, examinan la probabilidad de automatización de 702 ocupaciones y su incidencia en la robotización del trabajo; según sus resultados, el $47 \%$ del empleo está en riesgo de ser automatizado o robotizado en las próximas dos décadas (el porcentaje corresponde a Estados Unidos). Desafíos como estos producen crisis del mundo que conocemos, pero a la vez son una oportunidad porque invitan a re-pensar el papel de la universidad de cara al futuro, no solo como una institución que proporciona programas académicos, sino como un escenario que propicia la dignidad humana de quienes egresan, permanecen y quieren ingresar, así como de la comunidad universitaria que conforma la universidad, en contextos de profundización de los impactos de la globalización y el modelo neoliberal.

\section{Conclusiones}

Para los participantes en el estudio, la dignidad humana significa conocer, ejercer, gozar, ampliar su valor como seres humanos, al mismo tiempo que disfrutar de garantías para el ejercicio de sus derechos humanos. La dignidad para ellos es sinónimo de respeto, autoestima, derecho, felicidad, libertad, equidad, autonomía, vivir bien, amar y moral. Además, la universidad como institución significa un escenario que propicia la dignidad humana, al permitirles desarrollar parte de su plan vital, adquirir conocimientos, desarrollar habilidades, capacidades, y descubrir potencialidades superando incertidumbre $y$ soledad.

La dignidad al asociarla con la universidad se presenta como una forma de estabilización ante la incertidumbre del mundo contemporáneo. La universidad es un contexto en el que la exposición a crisis existenciales, angustias, incertidumbres y presiones ocasionadas por expectativas diversas, pueden hacer que los estudiantes presenten problemas de diferentes órdenes. Sin embargo, el estudiante al sentirse valorado, respetado, tiene mejores posibilidades de ejercer sus derechos, su autonomía, tener una autoimagen positiva, y de esta manera superar los problemas que se le presenten y enfrentar la incertidumbre.

Para este grupo de estudiantes, la dignidad es vivir bien, sin humillaciones, con posibilidades de desarrollar un plan vital, satisfacer deseos, encontrar la mejor manera de ser feliz, que para la mayoría de ellos, está en parte, en la oportunidad que brinda la universidad como institución pública de fortalecerlos como seres humanos integrales.

Este tipo de investigaciones permite a los participantes reconocimiento y dignificación al escuchar, discutir y socializar los diferentes significados atribuidos a la dignidad humana en relación con la universidad y ampliar y co-construir 
representaciones de lo que constituiría la dignidad humana.

Dada la complejidad del tema, las preguntas siguen abiertas: ¿qué es la dignidad humana, para las comunidades en concreto?, ¿por qué frente a la opresión muchas comunidades tienen como bandera la dignidad y como horizonte los derechos humanos?, ¿es la universidad una institución que permite la realización de la dignidad humana?

\section{Agradecimientos}

El autor agradece a los participantes por sus contribuciones, tiempo de trabajo y planteamientos.

\section{Referencias}

Berger, P. (1997). El pluralismo y la dialéctica de la incertidumbre. Estudios Públicos, 67, 1-18.

Carrillo, L. B. (2011). Espíritu universitario y autonomía. Revista De Lenguas Modernas, 14, 916.

Comunidad Andina de Naciones. (2013). Il Estudio Epidemiológico Andino sobre Consumo de Drogas en la Población Universitaria Informe Colombia, 2012. Lima-Perú.

Frey, C. B., \& Osborne, M. A. (2013). The future of employment: how susceptible are jobs to computerisation?. Oxford Martin School, Programme on the Impacts of Future Technology, University of Oxford. Recuperado de: Recuperado de: http://www.oxfordmartin.ox.ac.uk/ downloads/academic/The_Future_of_Employme nt.pdf

Foucault, M. (1978). Vigilar y castigar: nacimiento de la prisión. España:Siglo XXI editores.
Giddens, A. (2000). Un mundo desbocado. Los efectos de la globalización en nuestras vidas. Madrid:Taurus.

Gómez Pavajeau, C. A. (2011). La dignidad de la persona como fundamento del orden jurídico. La teoría del sujeto de derecho penal. Revista Derecho Penal y Criminología, 32(93), 27-42.

Habermas, J. (2010). El concepto de dignidad humana y la utopía realista de los derechos humanos. Diánoia, 55(64), 3-25.

Hernández, E. (2007) “Informe final diagnóstico de necesidades que en materia de salud mental presenta la comunidad universitaria. Proyecto: "Promoción y desarrollo del Bienestar Universitario en la sede Bogotá" Bogotá. Universidad Nacional de Colombia.

Herrera, J. (2008). La complejidad de los derechos humanos. Bases teóricas para una definición crítica. Revista Internacional de Direito e Cidadania, 1, 103-135.

Hobsbawm, E. J. (1995). Historia del siglo XX. 19141991. Región y Sociedad, 11(17.1999), 188.

Kant, I. (1989). Metafísica de las Costumbres, Segunda parte. Principios de la doctrina de la virtud, Madrid:Tecnos.

Kant, I. (2003). Fundamentación de la metafísica de las costumbres. México: Porrua.

Krotz, E. (2003). El multiverso cultural como laboratorio de vida feliz. Alteridades, 13(25), 35-44.

León, C. G. (2005). Política, ética y justicia social en la educación superior neoliberal. Bogota D.C: Universidad de los Andes. 
Levitas, R. (2007). Florecimiento humano: ¿una agenda utopista?.Desacatos, 23, 87-100.

Luhmann, N., Giddens, A., Bauman, N., \& Beck, U. (1996). En Las consecuencias perversas de la modernidad: modernidad, contingencia y riesgo. Barcelona: Anthropos.

Machado, J. (2001). La Universidad saludable. Revista de Ciencias Humanas, 7(26). Recuperado de: http://www.utp.edu.co/ chumanas/revistas/ revistas/rev26/machado.htm

Salamanca, A.B., \& Martín-Crespo, M. C. (Marzo, 2007). El muestreo en la investigación cualitativa. Nure Investigación (27). Recuperado de: http://www.nureinvestigacion.es/ficheros_admin istrador/f_metodologica/fmetodologica_27.pdf

Mendoza, M. L. (13 de marzo, 2014). "El neoliberalismo tomó por asalto a las universidades": Noam Chomsky. Periódico El
Espectador, sección Educación, Bogotá-Colombia. Recuperado de: http://www.elespectador.com/ noticias/educacion/el-neoliberalismo-tomoasalto-universidades-noam-chomsk-articulo480438.

Parra, R. (1994). La Soledad del Estudiante Universitario. En: Antia, M., Castro, M., Castro, L., Cruz, L., Ortiz, M., Oviedo, L, Franco, A., Ruiz, m., Rojas, L., \& Silva, E. La Universidad Adolescente. Universidad del Tolima. Colombia: Fondo Resurgir - FES.

Restrepo, M. (2010). Teoría crítica de los derechos humanos. Tunja: Universidad Pedagogica y Tecnológica de Colombia.

Sánchez, A. (2006). La juventud entre la historia y la sociología. Pap.polit. (11)2,797-806.

Sarmiento, L. (2013). Teoría Crítica Fundamento de los Derechos Humanos. Bogotá: LSA editores. 